\title{
Prediction of Hemorrhagic Transformation and Functional Prognosis in Patients With Acute Ischemic Stroke Using One-stop CT Examination: A Study on the Plateau Region of China
}

\section{Meng Li}

Affiliated Hospital of Qinghai University

Huiying $\mathrm{Hu}$

Affiliated Hospital of Qinghai University

Weixia Li

Affiliated Hospital of Qinghai University

Chengpin Bai

Affiliated Hospital of Qinghai University

\section{Xin Bai}

Affiliated Hospital of Qinghai University

\section{Xueyan Wang}

Affiliated Hospital of Qinghai University

\section{Yousen Wu}

Affiliated Hospital of Qinghai University

Guangshun Lu

Affiliated Hospital of Qinghai University

Haihua Bao ( $\nabla$ baohelen2@sina.com )

Affiliated Hospital of Qinghai University

\section{Research Article}

Keywords: hemorrhagic transformation, functional outcomes, MTT, Tmax, PS, rCBV, rMTT, rPS, HT, CTA

Posted Date: September 7th, 2021

DOI: https://doi.org/10.21203/rs.3.rs-839065/v1

License: (c) (1) This work is licensed under a Creative Commons Attribution 4.0 International License. Read Full License 


\section{Abstract}

There are few studies on the early hemorrhagic transformation of ischemic stroke in plateau area. This study aimed to analyze the value of one-stop CT in predicting HT and functional outcomes in patients with acute ischemic stroke (AIS) caused by occlusion of great vessels before treatment in the high plateau region. Data were obtained from patients who underwent non-enhanced CT (NCCT), CTP and CTA examination within 24 hours from October 2019 to December 2020. Follow-up CT/MRI was performed within one month to determine if HT occurred later. The clinical data, laboratory results and imaging data of patients with and without HT were compared. Of the 74 patients included, 32(43.3\%) had HT during follow-up, and HT was more likely to occur in poor collateral circulation $(p=0.029)$. The values of MTT, Tmax, PS, rCBV, rMTT, rPS in HT group were higher than those in non-HT group $(p<0.05)$. The results showed that PS > 1.315 ml/100 g/min (AUC, 0.753, $p=0.001$ ) and rCBV > 1.470(AUC, 0.764; $p=0.001$ ) had better diagnostic value for HT. One-stop CT examination was performed before treatment in patients with AIS. Quantitative perfusion parameters and multi-phase CTA were used to evaluate the prognosis of $\mathrm{HT}$ after recanalization of proximal great vessels.

\section{Introduction}

Ischemic stroke is a neurological disorder with high disability rate, high mortality rate and high incidence of complications. The transformation of intracranial hemorrhage is a serious complication after cerebral infarction or thrombolytic therapy ${ }^{[1]}$. After cerebral infarction, due to the blood vessels in the ischemic area reperfusing, the abnormal permeability of blood-brain barrier and the dysfunction of blood vessel basement membrane in the infarcted area may lead to the deterioration of nerve function, the incidence is about $10 \%-40 \%{ }^{[2]}$.At present, hyperacute therapy for acute ischemic stroke mainly includes intravenous thrombolysis and intravascular interventional therapy. Reperfusion therapy is helpful to improve the clinical outcome of acute ischemic stroke(AIS), reperfusion therapy increased the frequency and severity of $\mathrm{HT}^{[3][4]}$. Recent studies have shown that endovascular intervention improves prognosis in patients with acute ischemic stroke caused by anterior circulation occlusion within 6-8 hours of onset, but the risk of $\mathrm{HT}$ has been increasing in recent years. Currently, the assessment of HT risk depends primarily on clinical status and neuroimaging ${ }^{[5-6]}$. One-stop CT examination (NCCT, CTP and CTA) are the main diagnostic methods for AIS. The destruction of blood-brain barrier (BBB) is a key component in the development of hemorrhagic transformation. Several studies have shown that good collateral circulation is associated with a lower HT risk, in part because good collateral circulation provides adequate circulation to brain endothelial cells, resulting in a smaller infarct volume ${ }^{[7]}$. On the other hand, due to the destruction of the blood-brain barrier, the leakage of contrast media into the extravascular space may indicate the impairment of the blood-brain barrier, more likely to evolve into $H T$, and acquisition of perfusion images may predict the presence of HT in acute ischemic stroke. The aim of this study was to investigate the relationship between HT and global cerebral perfusion parameters, multiphase-CTA scores, and different treatments in patients with AIS. 


\section{Methods}

Statement.

This study received ethics approval by the ethics of the Affiliated Hospital of Qinghai University (AFRHEC-0062-01). Each patient included in this study received written information and informed consent was obtained from all the participants. This research study was in accordance with the Declaration of Helsinski. All studies were performed under all relevant guidelines and regulations.

\section{Patients.}

The data were from October 2019 to December 2020. The clinical and imaging data of AIS patients with acute neurological impairment within 12 hours were analyzed by whole brain volume CT perfusion and CT angiography. Inclusion criteria: (1) one-stop CT scan within 12 hours of onset in our stroke center; (2) diagnosis of anterior circulation infarction based on imaging; (3) patients who underwent CT scan again within one week after clinical diagnosis after treatment (including conservative therapy, intravenous thrombolysis, intravascular therapy). (4) patients older than 18 years and living at 2000-2500 m elevation; (5) the image quality is good and there is no obvious motion artifact. Exclusion Criteria: (1) unable to undergo one-stop stroke screening due to kidney failure, sensitivity to contrast agents, or other reasons; (2) patients with malignant tumors; (3) patients with mental illness; and (4) patients who refused follow-up.

Imaging Protocol.

All patients were scanned on the 128 - slice multidetector CT scanner(Discovery CT 750 HD; GE Medical Systems, Milwaukee, Wis). The scanning parameters were as follows: tube voltage $120 \mathrm{kv}$, tube current $300 \mathrm{~mA}$, scanning thickness $5 \mathrm{~mm}$, rotation time $1 \mathrm{~s}$ /circle; Using 4D spiral adaptive scanning (volume shuttle mode) to acquire whole brain perfusion CT data, continuous acquisition 28 times, the total scanning time is $47.38 \mathrm{~s}$, the tube voltage is $100 \mathrm{kv}$, the tube current is $80 \mathrm{~mA}$; the scanning thickness is $5 \mathrm{~mm}$, the time resolution is $1.6 \mathrm{~s}$; the pitch 0.984 , rotation speed $0.4 \mathrm{~s} /$ circle, field of view $320 \times 320 \mathrm{~mm}$; matrix $512 \times 512$.

Contrast agent injection scheme: $50 \mathrm{ml}$ iopromide $(370 \mathrm{mgl} / \mathrm{s})$ was injected at a flow rate of $4.5 \mathrm{ml} / \mathrm{s}$ by high-pressure mechanical injector and $30 \mathrm{ml}$ normal saline was injected at the same flow rate for scanning. After injection of iodine contrast agent, perfusion scanning began after $5 \mathrm{~s}$ delay. Three-phase CTA was performed $5 \mathrm{~min}$ later, and $50 \mathrm{~mL}$ iopromide $(370 \mathrm{mgl} / \mathrm{s}$ ) was injected with a high-pressure injection at the injection rate of $4.5 \mathrm{~mL} / \mathrm{s}$. The first phase was the peak period of the head and neck artery. The scanning range was from the aortic arch to the parietal skull (Tube voltage $100 \mathrm{Kv}$, tube current $200 \mathrm{~mA}$, rotating speed $0.5 \mathrm{~s} /$ cycle), scanning layer thickness $5 \mathrm{~mm}$, reconstruction layer thickness $0.625 \mathrm{~mm}$. The second phase was the peak period of the cranial vein, and the scanning was delayed by $8 \mathrm{~s}$ after the first phase scanning. The scanning range was from the skull base to the top of 
the skull. Phase 3 was the late stage of the cranial vein, which was delayed for $8 \mathrm{~s}$ after phase 2 scanning.

Contrast agent injection protocol: Inject $50 \mathrm{ml}$ of non-ionic contrast agent iopromide $(370 \mathrm{mgl} / \mathrm{s})$ through a high-pressure syringe at an injection flow rate of $4.5 \mathrm{ml} / \mathrm{s}$ and then inject $30 \mathrm{ml}$ of normal saline at the same flow rate for scanning. The perfusion scan was started after a delay of 5 s after the injection of iodine contrast agent; after 5 minutes, a three-phase CTA examination was performed, and $50 \mathrm{ml}$ of nonionic contrast agent iopromide $(370 \mathrm{mgl} / \mathrm{s})$ was injected through a high-pressure syringe at an injection flow rate of $4.5 \mathrm{ml} / \mathrm{s}$. The first phase is the peak period of the head and neck arteries. The scanning range is from the aortic arch to the top of the skull. The scanning technology is used to automatically track and trigger the contrast agent. The monitoring level is the aortic arch level. The monitoring starts 10 s after the injection of the iodine contrast agent. The threshold is set to $70 \mathrm{HU}$; tube voltage, $100 \mathrm{KV}$; tube current $200 \mathrm{~mA}$, rotation speed, $0.5 \mathrm{~s} /$ circle; scanning layer thickness, $5 \mathrm{~mm}$; reconstruction layer thickness, $0.625 \mathrm{~mm}$ ). The second phase is the peak period of cranial veins, after the first stage scan, the scan is delayed by $8 \mathrm{~s}$, and the scan range is from the base of the skull to the top of the skull; the third stage is the late stage of the cranial veins, and the scan is delayed $8 \mathrm{~s}$ after the second scan.

MRI data were collected by 3.0T magnetic resonance scanner (Magnetom Prisma, Siemens Healthineer) and 64 channel head coil. Conventional sequence scanning of head MR was performed: axial TIWI, T2WI, T2FLAIR and DWI. The specific scanning parameters were as follows:

TIWI: TR 150ms, TE 2.5ms, FOV 230mm, slice thickness $5.0 \mathrm{~mm}$.

T2Wl: TR 5000ms, TE $117 \mathrm{~ms}$, FOV 230mm, slice thickness 5.0mm.

DWI: TR 3230ms, TE 65ms, FOV 230mm, slice thickness $5.0 \mathrm{~mm}$.

T2 FLAIR: TR $8000 \mathrm{~ms}$, TE $81 \mathrm{~ms}$, FOV 230mm, layer thickness $5.0 \mathrm{~mm}$.

Image Reconstruction and Analysis

All data post-processing is carried out in AW4.7 software. Peak arterial phase and peak venous phase were selected on the two time-density curves. The input artery and output vein were contralateral middle cerebral artery and superior sagittal sinus. Selecting the largest and most obvious abnormal perfusion level on the image of TTP to describe the region of interest (ROI) manually, CBV, CBF), TTP, MTT, Tmax and PS were collected automatically. The rCBF, rCBV, rMTT, rTTP, rTmax, rPS were calculated on the contralateral side as the normal control area. The ischemic core volume was defined as $\mathrm{rCBF}<30 \%{ }^{[8-9]}$. Low perfusion volume is defined as the volume of $\operatorname{Tmax}>6 \mathrm{~s}^{[9-10]}$, which is calculated automatically by software. Fast-stroke software (GE Healthcare, Milwaukee, WI, USA) was used for automatic analysis of multi-phase CTA data, including arterial peak period and MIP image acquisition. According to the European cooperative acute stroke study (ECASS), patients were divided into HT and non-HT groups. The main outcome was a modified Rankin score ${ }^{[8]}$ at 90 days, which defined good outcomes as $0-2$ and poor 
outcomes as 3-6. The side branch score of mCTA was Bijoy K. Menon ${ }^{[9]}$ and others put forward $0-5$ score method, defining $0-2$ as poor collateral circulation, 3-5 as good collateral circulation.

\section{Statistical Analysis}

Statistical analysis using SPSS25.0 software, Normality test and variance homogeneity test, consistent with the normal distribution using independent sample t test, skewness distribution using Wilcoxon rank sum test. The counting data were measured by chi-square test or exact probability method. The receiver operating characteristic $(\mathrm{ROC})$ curve was used to assess the different risk factors for predicting $\mathrm{HT}$. The test level was bilateral $a=0.05$, with $P<0.05$, the difference was statistically significant.

\section{Results}

\subsection{Population Baseline}

Seventy-four patients (male, 50 ; females, 24 ; average age of $64.42 \pm 13.05$ years) were finally enrolled. Of these patients, $70.3 \%$ had right hemisphere infarction, 42 had hypertension, 17 had diabetes, 7 had atrial fibrillation and 11 had coronary heart disease. There were 24 cases $(32.4 \%)$ with intracranial internal carotid artery occlusion, 43 cases (58.1\%) with middle cerebral artery occlusion (M1/M2), and 7 cases (9.5\%) with both $\mathrm{M} 1 / \mathrm{M} 2$ occlusion. 37 patients (50\%) received conservative treatment, 23 patients $(31.1 \%)$ received intravenous thrombolytic therapy, and 14 patients $(18.9 \%)$ received intravascular therapy. In all patients, 32(43.2\%) had HT, and the baseline NIHSS score in HT group was higher than that in non-HT group $(p=0.036)$. The counts of white blood cells, red blood cells and platelets in HT group and non-HT group were not significant $(P>0.05)$. (Table 1,Fig. 1) 
Table 1

Baseline clinical data.

\begin{tabular}{|c|c|c|c|}
\hline & HT $(n=32)$ & Non-HT $(n=42)$ & $\mathbf{P}$ \\
\hline Age (years), mean $\pm S D$ & $67.03 \pm 12.90$ & $62.43 \pm 12.97$ & .134 \\
\hline Male(\%) & 21(34.4) & $29(69.0)$ & .755 \\
\hline Infarct site (right) ,n(\%) & $25(78.1)$ & $27(64.3)$ & .197 \\
\hline NIHSS, median (IQR) & $8.50(4.00-12.75)$ & $6(3.00-11.00)$ & .036 \\
\hline \multicolumn{4}{|l|}{ Risk factors, n (\%) } \\
\hline Hypertension & 18(56.3) & $24(57.1)$ & .939 \\
\hline Diabetes mellitus & $6(18.8)$ & $11(26.2)$ & .451 \\
\hline Atrial fibrillation & $4(12.5)$ & $3(7.1)$ & .457 \\
\hline Smoking & 11(34.4) & 19(45.2) & .346 \\
\hline Drinking & $9(28.1)$ & $12(28.6)$ & .996 \\
\hline Coronary heart disease & $6(18.8)$ & $5(11.9)$ & .412 \\
\hline \multicolumn{4}{|l|}{ Laboratory parameters } \\
\hline $\mathrm{WBC} \times 10^{9}$ cells $\left./ \mathrm{L}\right)$, median (IQR) & 7.99(6.44-10.49) & 7.34(5.09-9.16) & .153 \\
\hline $\begin{array}{l}\text { RBC (×1012cells/L), } \\
\text { median (IQR) }\end{array}$ & $4.79(4.40-5.73)$ & $5.20(4.60-5.38)$ & .487 \\
\hline $\begin{array}{l}\text { Platelet count }(\times 109 \\
\text { cells/L), mean } \pm \text { SD }\end{array}$ & $158.50(127.50-190.25)$ & $158.00(142.50-189.50)$ & .545 \\
\hline PT & 11.45(10.78-12.23) & $11.20(10.15-11.55)$ & .133 \\
\hline APTT & $25.55(22.8-30.60)$ & $25.35(22.90-28.68)$ & .532 \\
\hline INR & $0.95(0.91-1.03)$ & $0.93(0.84-0.97)$ & .193 \\
\hline Occlusion site,n(\%) & & & .879 \\
\hline ICA & $9(28.1)$ & $15(35.7)$ & \\
\hline MCA & $20(62.5)$ & $23(54.8)$ & \\
\hline $\begin{array}{l}\text { Tandem occlusion (ICA1 } \\
\text { MCA M1/M2) }\end{array}$ & $3(9.4)$ & $4(9.5)$ & \\
\hline Treatment methods & & & .594 \\
\hline
\end{tabular}




\begin{tabular}{|lll|}
\hline & HT $(\mathbf{n}=\mathbf{3 2})$ & Non-HT $(\mathbf{n}=42) \quad P$ \\
\hline Conservative treatment & $17(53.1)$ & $20(47.6)$ \\
\hline Thrombolysis & $8(25.0)$ & $15(35.7)$ \\
\hline Endovascular treatment & $7(21.9)$ & $7(16.7)$ \\
\hline
\end{tabular}

Fig 1 A 77-year-old woman presented with right limb weakness and slurred speech within 2 hours. Images showed decreased CBF values(A) in the left temporal lobe, TTP(B), Tmax(C), and MTT(D) values prolonged, and left middle cerebral artery acute ischemic stroke hypoperfusion. PS(E) shows abnormal pseudo-color. The CTA (F) shows a left MCA M2 occlusion. The maximum intensity projection images $(\mathrm{G}, \mathrm{H}, \mathrm{I})$ of mCTA showed that the ischemic region delayed one phase and the number of vessels increased compared with the contralateral hemisphere. These results indicate a score of 4 . Follow-up MRI $(J)$ showed left paraventricular hemorrhage 24 hours after intravenous thrombolytic therapy.

3.2 Comparison of multi-phase CTA scores between HT Group and non-HT group

Of the 74 patients, 2 cases ( $2.7 \%$ ) scored 0,6 cases (8.1\%)scored 1, 23 cases(31.1\%) scored 2, 17 cases $₫ 23.0 \% \llbracket$ scored 3,19 cases $₫ 25.7 \% \llbracket$ scored 4 and 7 cases $\varangle 9.5 \%$ scored 5 . Among 31 patients with mCTA, 18(58.1\%) had HT, 43 had good collateral circulation, and 14(32.6\%) had HT. There was significant difference in the incidence of HT between the two groups $(p<0.05)$. (Table 2$)$ 
Table 2

Imaging characteristics.

\begin{tabular}{|llll|}
\hline & HT(n= 32) & Non-HT(n= 42) & P \\
\hline Time (Onset to imaging), (median) (IQR) & 295.00 & 353.00 & .192 \\
& $(154.25-456.52)$ & $(234.75-505.75)$ & \\
\hline Treatment methods & & & .594 \\
\hline Conservative treatment & $17(53.1)$ & $20(47.6)$ & \\
\hline Thrombolysis & $8(25.0)$ & $15(35.7)$ & \\
\hline Endovascular treatment & $7(21.9)$ & $7(16.7)$ & .066 \\
\hline Ischemic core volume,ml, (median) (IQR) & 17.82 & 15.22 & .655 \\
\hline Hypoperfusion volume,ml, (median) (IQR) & 88.17 & $(9.48-24.22)$ & \\
\hline Final infarct volume,ml, (median) (IQR) & 38.96 & 90.57 & .010 \\
\hline Poor outcome at 3 months, $n$ (\%) & $(31.26-42.31)$ & $(29.92-38.32)$ & \\
\hline Poor collateral circulation, $n$ (\%) & $25(78.1)$ & $20(47.6)$ & .008 \\
\hline
\end{tabular}

3.3 Comparison of perfusion parameters between HT group and non-HT group

The values of MTT, Tmax, PS, rCBV, rMTT, rPS in HT group were higher than those in non-HT group $(\mathrm{p}<$ 0.05). There was no significant difference in CBV, CBF, TTP, RCBF, rTTP and rTmax between the two groups $(P>0.05)$ (Table 3). PS, rPS, rCBV, Tmax and MTT were included in the diagnostic efficacy analysis. PS (AUC, 0.753, [ 95\%Cl] : 0.63,0.87; P, 0.001) and rCBV (AUC, 0.764; [95\% Cl] : 0.64,0.89; P, 0.001 ) had better diagnostic efficacy for HT (Fig. 1, Table 4). The results showed that rCBV value was more than 1.470, PS value was more than 1.315, rPS value was more than 2.880 , Tmax value was more than 13.82, MTT value was more than 13.45, rMTT value was more than 4.315, which was the best critical value, can be used to identify patients with hemorrhagic transformation.(Table 4,Fig. 2) 
Table 3

Data of perfusion parameters.

\begin{tabular}{|llll|}
\hline Perfusion parameters & $\mathrm{HT}(\mathbf{n}=\mathbf{3 2})$ & Non-HT(n=42) & $\mathbf{P}$ \\
\hline $\mathrm{CBV}(\mathrm{ml} / 100 \mathrm{~g})$ & $3.32 \pm 3.17$ & $2.46 \pm 0.87$ & .230 \\
\hline $\mathrm{CBF}\left(\mathrm{ml} \cdot 100 \mathrm{~g}^{-1} \cdot \mathrm{min}^{-1}\right)$ & $19.00 \pm 51.54$ & $13.57 \pm 11.27$ & .144 \\
\hline $\mathrm{MTT}(\mathrm{s})$ & $22.20 \pm 7.99$ & $18.65 \pm 9.94$ & .044 \\
\hline $\mathrm{TTP}(\mathrm{s})$ & $23.96 \pm 7.42$ & $21.84 \pm 7.19$ & .181 \\
\hline Tmax $(\mathrm{s})$ & $16.79 \pm 7.78$ & $12.25 \pm 7.40$ & .022 \\
\hline PS $\left(\mathrm{ml} \cdot 100 \mathrm{~g}^{-1} \cdot \mathrm{min}^{-1}\right)$ & $1.94 \pm 1.53$ & $0.83 \pm 0.78$ & .000 \\
\hline rCBV & $1.93 \pm 1.74$ & $1.00 \pm 0.43$ & .000 \\
\hline rCBF & $0.52 \pm 0.64$ & $0.52 \pm 0.41$ & .243 \\
\hline rMTT & $3.97 \pm 2.39$ & $2.89 \pm 1.62$ & .037 \\
\hline rTTP & $1.73 \pm 0.58$ & $1.66 \pm 0.61$ & .719 \\
\hline rTmax & $4.35 \pm 3.29$ & $3.58 \pm 2.38$ & .345 \\
\hline rPS & $4.61 \pm 4.02$ & $2.81 \pm 1.58$ & .012 \\
\hline
\end{tabular}

Table 4

Efficacy analysis of the perfusion parameters.

\begin{tabular}{|c|c|c|c|c|c|c|c|c|}
\hline & AUC & $95 \% \mathrm{Cl}$ & $\mathbf{P}$ & Sensitivity & $95 \% \mathrm{Cl}$ & Specificity & $95 \% \mathrm{Cl}$ & Threshold \\
\hline rCBV & 0.764 & $\begin{array}{l}0.6413 \text { to } \\
0.8870\end{array}$ & 0.000 & 0.500 & $\begin{array}{l}33.63- \\
66.37 \%\end{array}$ & 95.24 & $\begin{array}{l}84.21- \\
99.15 \%\end{array}$ & $>1.470$ \\
\hline PS & 0.753 & $\begin{array}{l}0.6322 \text { to } \\
0.8745\end{array}$ & 0.000 & 0.625 & $\begin{array}{l}45.25- \\
77.07 \%\end{array}$ & 0.905 & $\begin{array}{l}77.93- \\
96.23 \%\end{array}$ & $>1.315$ \\
\hline rPS & 0.672 & $\begin{array}{l}0.5467 \text { to } \\
0.7970\end{array}$ & 0.012 & 0.625 & $\begin{array}{l}45.25- \\
77.07 \%\end{array}$ & 0.683 & $\begin{array}{l}53.02- \\
80.44 \%\end{array}$ & $>2.880$ \\
\hline Tmax & 0.656 & $\begin{array}{l}0.5324 \text { to } \\
0.7801\end{array}$ & 0.022 & 0.594 & $\begin{array}{l}42.26- \\
74.48 \%\end{array}$ & 0.6905 & $\begin{array}{l}53.97- \\
80.93 \%\end{array}$ & $>13.82$ \\
\hline MTT & 0.637 & $\begin{array}{l}0.5114 \text { to } \\
0.7631\end{array}$ & 0.044 & 0.969 & $\begin{array}{l}84.26- \\
99.84 \%\end{array}$ & 0.381 & $\begin{array}{l}25.00- \\
53.19 \%\end{array}$ & $>13.45$ \\
\hline rMTT & 0.642 & $\begin{array}{l}0.5140 \text { to } \\
0.7702\end{array}$ & 0.037 & 0.344 & $\begin{array}{l}20.41- \\
51.69 \%\end{array}$ & 0.905 & $\begin{array}{l}77.93- \\
96.23 \%\end{array}$ & $>4.315$ \\
\hline
\end{tabular}


Hemorrhagic transformation $(\mathrm{HT})$ in cerebral infarction leads to increased mortality or poor functional recovery. Recanalization can increase the risk of $\mathrm{HT}$. The rate of symptomatic intracerebral hemorrhage in rtPA was about $1.6 \%-4.8 \%$ and the mortality was $45 \%$ in the large stroke thrombolytic registry ${ }^{[10-11]}$. The abnormal permeability of the blood brain barrier (BBB) caused by capillary endothelial dysfunction in the infarcted area after ischemic stroke is the basis of cerebral edema and hemorrhagic transformation of ischemic brain tissue, could cause blood to leak out ${ }^{[12-13]}$. CTP estimates the vascular permeability of contrast media by prolonging the acquisition time, and the blood brain barrier (BBB) injury can be quantified by measuring vascular permeability. The vascular surface permeability (PS) in CT perfusion parameters reflects the permeability of the blood brain barrier. The ratio of PS to healthy PS in ischemic stroke patients is called rPS. With the increase of the ratio, the difference of the damage degree of bloodbrain barrier is bigger ${ }^{[14]}$. Evaluation of blood-brain barrier permeability with these perfusion parameters may make possible early prediction of hemorrhagic transformation. Our results confirmed that PS in the ischemic region was higher than that in the contralateral non-ischemic tissue. When the optimal threshold of PS was 1.315, the sensitivity of predicting HT in ischemic brain tissue was $62.5 \%$ (95\% Cl: 45.25\%-77.07\%), specificity was $90.5 \%$ (95\% Cl: $77.93 \%-96.23 \%$ ). When the optimal threshold of rPS was 2.880, the sensitivity and specificity of predicting HT were $62.5 \%$ (95\% Cl: $45.25 \%-77.07 \%$ ) and $68.3 \%$ (95\% Cl: $53.02 \%-80.44 \%$ ) respectively. This is consistent with the results of Li et al ${ }^{[14]}$. It shows that PS and rPS have high value in predicting HT. In our study, the specificity of PS optimal threshold was low, only $68.3 \%$. The reason may be that PS reflects the permeability of the blood-brain barrier around the necrotic area rather than within the necrotic area, the necrotic area is the preferred site for HT, as the necrotic tissue is the most severely damaged and therefore the highest risk of bleeding ${ }^{[15]}$. In order not to overestimate the risk of $\mathrm{HT}$, it is necessary to analyze it in conjunction with other clinical and radiological parameters. In previous studies, Yassi et al. ${ }^{[11]}$ concluded that Tmax $>14 \mathrm{~s}$ and volume $>5 \mathrm{~mL}$ were associated with substantial hematoma, and that the rMTT median was longer in HT patients than in nonHT patients, the value of Tmax was prolonged (16.79 \pm 7.78 vs $12.25 \pm 7.40)$ and the value of rTmax was prolonged ( $4.35 \pm 3.29$ vs $3.58 \pm 2.38$ ). In addition, Jain ${ }^{[16]}$ found that rCBV was the strongest predictor of HT in his study. The optimal threshold of rCBV was 0.98 , and the specificity was $72 \%$. In our study, the area under the rCBV ROC curve was $0.764(p=0.000,95 \% \mathrm{Cl}, 0.64-0.89)$, the optimal threshold of RCBV was 1.470 , and the specificity was $95.24 \%$, which was higher than the optimal threshold suggested by Jain et al ${ }^{[16]}$. In recent years, Ozkul et al ${ }^{[15]}$ have demonstrated that $\mathrm{ICBV}$ is a reliable parameter for identifying ischemic penumbra in AIS. In other words, penumbra viability is an indirect predictor of HT. In the early stage of acute ischemia, the blood perfusion pressure of ischemic penumbra around infarction fluctuates in a certain range, $\mathrm{CBV}$ is relatively stable and $\mathrm{CBF}$ is slightly decreased by compensation of collateral circulation, MTT, TTP and Tmax are prolonged, if ischemia continues to worsen, CBF and CBV were decreased, and irreversible cerebral infarction occurred. We found a correlation between the presence of HT and poor collateral circulation. Multiphasic CTA in this study showed that $58.1 \%$ of patients with poor collateral circulation developed $\mathrm{HT}$, while $32.6 \%$ of patients with good collateral circulation developed HT. Previous studies have shown that the establishment of good collateral circulation, the lower the risk of developing $\mathrm{HT}^{[7][17]}$. Previous studies ${ }^{[18-19]}$ have also found that 
collateral circulation plays a decisive role in the volume of the infarct core and the size of ischemic tissue, the viability of the hypoperfusion brain tissue is maintained and the rate of infarction is slowed. The improvement of collateral circulation around cerebral ischemia area is related to better recanalization rate, lower hemorrhagic conversion rate and clinical prognosis after thrombolytic therapy ${ }^{[20]}$.Because HT can lead to clinical deterioration and related adverse outcomes by increasing brain tissue injury, therapeutic interventions that may aggravate HT have important implications, as explained in previous

studies $^{[2]}$. In our study, no association was found between thrombolysis or endovascular therapy and the development of HT. This difference may be due to the small number of patients in each group and the delay in follow-up imaging. The location of image judgment, the time and type of follow-up imaging required, the method of evaluation and classification of clinical deterioration, and the way in which imaging and clinical deterioration are performed vary from study to study and definition ${ }^{[21]}$, it is difficult to accurately assess the relationship between CTP parameters in an ischemic region and postoperative $\mathrm{HT}$ when multiple risk factors for HT are present, in order to obtain higher reliability, the correlation and restriction between these parameters are existed, and the optimal parameter threshold value of these parameters is determined, combining WCP parameters with multi-temporal CTA score can improve the ability of predicting $\mathrm{HT}$ to some extent, which may help clinicians to identify high-risk patients and make better treatment decisions. It will facilitate the study of measures to reduce the frequency, severity and clinical outcome of HT in patients with acute ischemic stroke.

Our study had several limitations. First, it was a single-center study. We did not analyze the subtypes to explore the differences in perfusion parameters between regions. In addition, the regions of interest for ischemic regions of the brain were mapped by an experienced operator and no attempt was made to assess reproducibility between different observers. In addition, we derive the threshold from the ROI analysis by including a parameter that may lead us to underestimate and overestimate the credibility of the metric, respectively.

\section{Conclusion}

One-stop CT examination was performed before treatment in patients with ischemic stroke. Quantitative perfusion parameters and multi-temporal CTA were used to evaluate the prognosis of $\mathrm{HT}$ after recanalization of proximal great vessels.

\section{Declarations}

\section{Acknowledgements}

This work was supported by Key Clinical Specialty Projects in Qinghai Province and Science and Technology Project of Qinghai Province (No. 2017-SF-158).

\section{Author contributions}


The main manuscript was written by Haihua Bao and Meng Li. Huiying Hu and Xueyan Wang scored the collateral circulation of the patients. Chengping Bai and Weixia Li collected the clinical data of the patients. Xin Bai, Guangshun Lu and Yousen Wu were responsible for were responsible for the image quality evaluation.

\section{Competing interests}

The authors declare no competing interests.

\section{References}

1. Lu, G. et al. Potential biomarkers for predicting hemorrhagic transformation of ischemic stroke[J]. Int J Neurosci, 128 (1), 79-89 (2018).

2. álvarez-Sabín. et al. Factors influencing haemorrhagic transformation in ischaemic stroke.[J]. Lancet Neurol, 12 (7), 689-705 (2013).

3. Prabhakaran. et al et al. Acute Stroke Intervention A Systematic Review[J] (Jama the Journal of the American Medical Association, 2015).

4. Balami, J. S. et al. Neurological complications of acute ischaemic stroke[J]. Lancet Neurol, 10 (4), 357-371 (2011).

5. Jovin, T. G. et al. Thrombectomy within 8 hours after symptom onset in ischemic stroke[J]. N Engl J Med, 372 (24), 2296-2306 (2015).

6. Saver, J. L. et al. Stent-retriever thrombectomy after intravenous t-PA vs. t-PA alone in stroke[J]. N Engl J Med, 372 (24), 2285-2295 (2015).

7. Li, X. et al. The Value of Whole-Brain Perfusion Parameters Combined with Multiphase Computed Tomography Angiography in Predicting Hemorrhagic Transformation in Ischemic Stroke[J]. J Stroke Cerebrovasc Dis, 29 (4), 104690 (2020).

8. Quinn, T. J. et al. Reliability of the modified Rankin Scale: a systematic review[J]., 40 (10), 33933395 (2009).

9. Menon, B. K. et al. Multiphase CT Angiography: A New Tool for the Imaging Triage of Patients with Acute Ischemic Stroke[J]., 275 (2), 510 (2015).

10. Mazya, M. et al. Predicting the risk of symptomatic intracerebral hemorrhage in ischemic stroke treated with intravenous alteplase: safe Implementation of Treatments in Stroke (SITS) symptomatic intracerebral hemorrhage risk score[J]., 43 (6), 1524-1531 (2012).

11. Yassi, N. et al. Prediction of poststroke hemorrhagic transformation using computed tomography perfusion[J]., 44 (11), 3039-3043 (2013).

12. Berkhemer, O. A. et al. A randomized trial of intraarterial treatment for acute ischemic stroke[J]. $N$ Engl J Med, 372 (1), 11-20 (2015). 
13. Van Kranendonk, K. R. et al. Hemorrhagic transformation is associated with poor functional outcome in patients with acute ischemic stroke due to a large vessel occlusion[J]. J Neurointerv Surg, 11 (5), 464-468 (2019).

14. Li, Q. et al. Permeability Surface of Deep Middle Cerebral Artery Territory on Computed Tomographic Perfusion Predicts Hemorrhagic Transformation After Stroke[J]., 48 (9), 2412-2418 (2017).

15. Ozkul-Wermester, O. et al. Increased blood-brain barrier permeability on perfusion computed tomography predicts hemorrhagic transformation in acute ischemic stroke[J]. Eur Neurol, 72 (1-2), 45-53 (2014).

16. Jain, A. R. et al. Association of CT perfusion parameters with hemorrhagic transformation in acute ischemic stroke[J]. AJNR Am J Neuroradiol, 34 (10), 1895-1900 (2013).

17. Ginsberg, M. D. et al. The cerebral collateral circulation: Relevance to pathophysiology and treatment of stroke[J]., 134 (Pt B), 280-292 (2018).

18. Campbell, B. C. et al. EXTEND-IA Investigators. Endovascular therapy for ischemic stroke with perfusion-imaging selection. N Engl J Med. 2015 Mar 12;372(11):1009-18.

19. Berkhemer, O. A. et al. Collateral Status on Baseline Computed Tomographic Angiography and IntraArterial Treatment Effect in Patients With Proxcimal Anterior Circulation Stroke[J]., 47 (3), 768-776 (2016).

20. Iryo, Y. et al. Collateral circulation via the circle of Willis in patients with carotid artery steno-occlusive disease: evaluation on 3-T 4D MRA using arterial spin labelling[J]. Clin Radiol, 70 (9), 960-965 (2015).

21. Aviv, R. I. et al. Hemorrhagic transformation of ischemic stroke: prediction with CT perfusion[J]., 250 (3), 867-877 (2009).

\section{Figures}

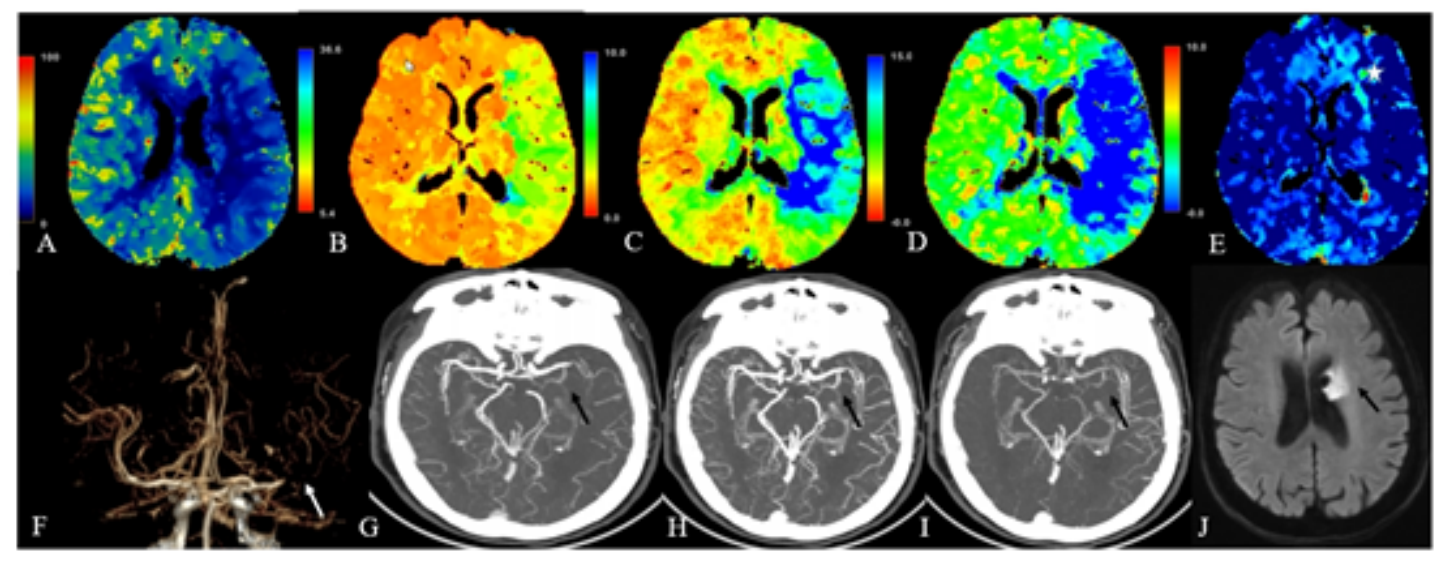

\section{Figure 1}

A 77-year-old woman presented with right limb weakness and slurred speech within 2 hours. Images showed decreased CBF values(A) in the left temporal lobe, TTP(B), Tmax(C), and MTT(D) values 
prolonged, and left middle cerebral artery acute ischemic stroke hypoperfusion. PS(E) shows abnormal pseudo-color. The CTA (F) shows a left MCA M2 occlusion. The maximum intensity projection images $(\mathrm{G}, \mathrm{H}, \mathrm{I})$ of mCTA showed that the ischemic region delayed one phase and the number of vessels increased compared with the contralateral hemisphere. These results indicate a score of 4 . Follow-up MRI $(J)$ showed left paraventricular hemorrhage 24 hours after intravenous thrombolytic therapy.

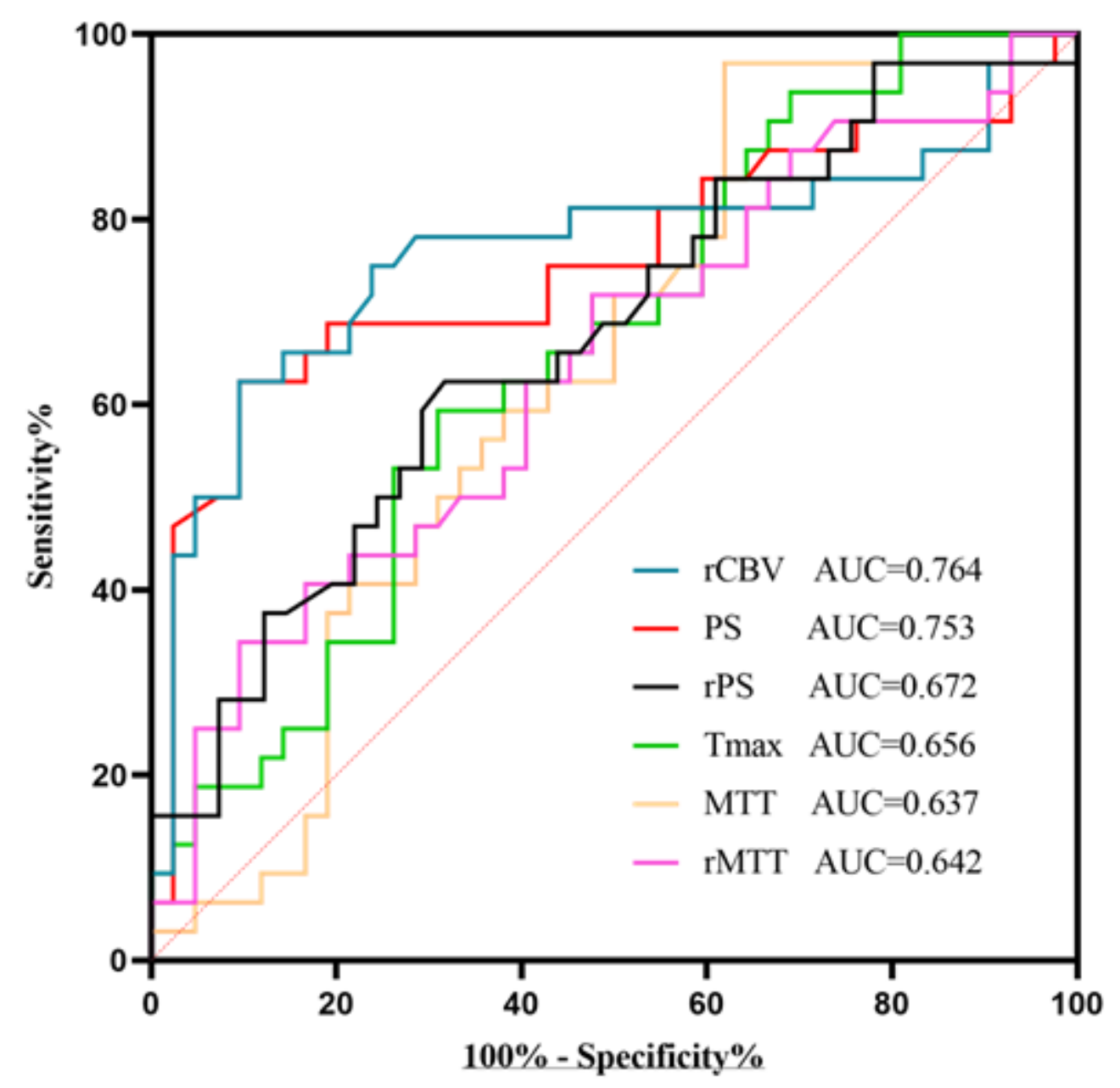

Figure 2

ROC curves of Efficacy analysis of the perfusion parameters 